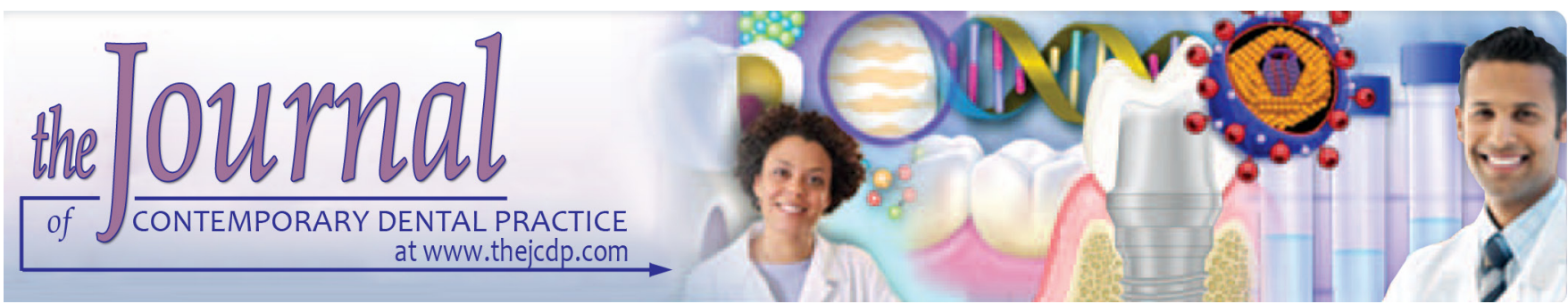

\title{
Toothbrushing-Should We Advise Mechanical or Power Brushes? Results of an International Delphi Conference
}

\author{
${ }^{1}$ Crawford Bain, ${ }^{2}$ Arwa Al Sayed, ${ }^{3}$ Eleftherios G Kaklamanos, ${ }^{4}$ Hizbullah AA Kazi
}

\begin{abstract}
Background and aim: To develop evidence-based answers to a series of questions in relation to toothbrushing using a modified Delphi consensus approach.

Oral hygiene and especially toothbrushing have been a fundamental part of the efforts to prevent caries and gingivitis. The questions discussed involved the frequency and duration of brushing, the effectiveness and safety of powered brushes and the recommendations for children and orthodontic patients.

Review results: The Delphi panel agreed that twice daily brushing for 2 minutes and a systematic pattern is advised. Moreover, it was concluded that evidence suggests that power brushes are safe and more effective in the short and long-term compared to manual brushes in terms of plaque removal and gingivitis reduction. For children and orthodontic patients, the likelihood of enhancing compliance/convenience with use of a power brush was highlighted.
\end{abstract}

Conclusion: Toothbrushing constitutes a fundamental part of the efforts to prevent caries and gingivitis.

Clinical significance: Twice daily brushing for 2 minutes and a systematic pattern is advised. Power brushes are safe and could provide benefits, for adults, children and orthodontic patients.

Keywords: Delphi consensus conference, Manual brushes, Power brushes, Toothbrushing

How to cite this article: Bain C, Sayed AA, Kaklamanos EG, Kazi HAA. Toothbrushing-Should We Advise Mechanical or

\footnotetext{
1,3 Hamdan Bin Mohammed College of Dental Medicine, Mohammed Bin Rashid University of Medicine and Health Sciences, Dubai, United Arab Emirates.

${ }^{2}$ Prince Sultan Medical Military City, Riyadh, Kingdom of Saudi Arabia.

${ }^{4}$ Procter and Gamble Gulf, Dubai, United Arab Emirates

Corresponding author: Eleftherios G Kaklamanos, Hamdan Bin Mohammed College of Dental Medicine (HBMCDM), Mohammed Bin Rashid University of Medicine and Health Sciences (MBRU), Building 34, Dubai Healthcare City, Dubai, United Arab Emirates Tel: +971 52969 1479, e-mail: eleftherios. kaklamanos@mbru.ac.ae; kaklamanos@yahoo.com
}

Power Brushes? Results of an International Delphi Conference. The Journal of Contemporary Dental Practice, October 2018;19(10):1169-1173.

\section{Source of support: $\mathrm{Ni}$}

Conflict of interest: Crawfor Bain, Arwa Al Sayed and Eleftherios G. Kaklamanos do not have any conflict of interest. Dr. Hizbullah Ashhad A. Kazi is the Professional and Scientific Relations Manager at Procter and Gamble Gulf.

\section{BACKGROUND AND AIM}

For over a century, and particularly since the role of dental plaque in the initiation of caries and gingivitis was identified, oral hygiene instruction has been considered a fundamental part of the efforts to prevent or minimize these diseases. An abundance of recent evidence indicates that failure to control inflammatory periodontal diseases has ramifications well beyond the oral cavity and can adversely affect the management of diabetes, cardiovascular diseases, and pregnancy. ${ }^{1}$

Most dental health professionals' advice to patients, still consists of twice daily toothbrushing with a fluoridecontaining toothpaste, flossing once or twice per day and regular use of other interproximal cleaning tools. Mouthwashes with antimicrobial properties and/or therapeutic levels of fluoride are also often recommended and are occasionally combined with irrigation devices.

Tooth brushing is the most widely used oral hygiene method. Systematic reviews have concluded that interproximal cleaning and particularly self-administered flossing has little clinical benefit in the prevention of caries, gingivitis or periodontitis. ${ }^{2,3}$ A Cochrane review $^{4}$ found a statistically but not clinically significant reduction in Gingival Index when comparing flossing and tooth brushing with toothbrushing alone. Current literature is however clear that tooth brushing is by far the most effective and patient-acceptable oral hygiene method. The aim of this Delphi study was to develop consensus on a series of toothbrushing related questions. 
A group of experts in various dental disciplines used a modified Delphi method to develop evidence-based answers to a series of questions in relation to toothbrushing. ${ }^{5}$ These statements had to have a strong evidence base where one was available, clearly identify areas where high-quality evidence could not be found, give an expert opinion where appropriate and recommend areas of necessary future research.

\section{REVIEW RESULTS}

\section{How Often should We Brush out Teeth?}

Kressin et al. ${ }^{6}$ found that consistent brushing (at least once daily) resulted in $49 \%$ reduction of the risk of tooth loss when compared to less frequent brushing. This longterm (26 years) study is one of very few using a true and not a surrogate outcome. Bosman and Powell ${ }^{7}$ showed that effective brushing once a day or every second day was sufficient to reverse gingivitis within 7 to 10 days while Lang et al. ${ }^{8}$ again showed that effective brushing once a day or every second day prevented all clinical signs of gingivitis over a 6-week period. There is no clear evidence of an additional benefit to brushing twice a day over once a day provided the cleaning is effective. Since it is impractical to expect a patient to reliably brush every second day, brushing effectively once a day appears to be both evidence-based and practical.

In high caries risk individuals, twice daily use of fluoride toothpaste has been shown to be more effective in caries reduction than less frequent brushing. It appears that the application of fluoride is more important than total plaque removal in achieving this outcome. The recommendations formed by the panel were the following:

- Brushing should be performed twice daily

- At least one of the two brushing sessions should last two minutes with a systematic pattern of effective tooth brushing. This should include all accessible tooth surfaces and could be considered a thorough clean; the second brushing is chiefly required to apply fluoride from the paste on the tooth surfaces as well as to freshen the breath.

- Recommendations for brushing frequency are also patient need dependent and may vary based on underlying pathology or conditions.

\section{How Long should We Brush for?}

It has been suggested that effective plaque removal in an intact dentition takes around 2 minutes, and that shorter periods result in less plaque removal and greater signs of inflammation. ${ }^{9}$ Shorter periods of brushing, even repeated several times a day, tend to miss the same areas each time and cannot be considered to be a thorough clean. Recommendations are therefore as follows:
1. A minimum of two minutes brushing is needed to maximize effective plaque removal.

2. There should be a systematic pattern of effective tooth brushing which includes all accessible tooth surfaces.

3. Special cases should get individualized brushing instructions to allow for optimum plaque removal.

\section{Are Powered Brushes more Effective than Manual Brushes?}

A recent Cochrane Collaboration systematic review ${ }^{10}$ looked at 51 trials involving 4624 participants, which provided data for meta-analysis. They found that powered brushes removed more plaque and reduced gingivitis more effectively than manual brushes in the short and long term. These results were modest but significant. Therefore, it was advised:

- Evidence suggests that power brushes are more effective in the short and long-term compared to manual brushes in terms of plaque removal and gingivitis reduction.

- According to present data, over the short and long term, power brushes may probably be better at maintaining oral health.

- Few studies exceed beyond six months duration. There is a necessity to conduct longer-term studies and ideally use real rather than surrogate outcome measurements.

\section{Is One Type of Powered Movement Superior?}

In the same Cochrane collaboration review, ${ }^{10}$ this time considering 27 studies the authors concluded that rotation-oscillation brushes are more effective in reducing plaque and gingivitis more than brushes with side-to-side actions. After the Delphi approach, it was concluded that:

- Present evidence suggests that oscillating-rotating power brushes are consistently more effective in reducing plaque and gingivitis compared to all others in the short- and long-term.

- Few studies exceed beyond 6 months duration. There is a necessity to conduct longer-term studies and ideally use real rather than surrogate outcome measurements.

\section{What should be Recommended to Children?}

Dental caries is the most significant cause of tooth loss in children. Gibson and Williams ${ }^{11}$ in a cross-sectional study of 1450 British preschool children conclude that regular brushing (twice a day) with fluoride toothpaste may have greater impact on caries in young children than restricting sugary foods. Thorough, twice daily 
application of fluoride via. toothpaste, combined with appropriate dietary control, is likely optimal in reducing and preventing caries in children. The recommendations of the expert panelists are as follows:

- Twice a day brushing is recommended.

- Children should be aided, until they develop adequate dexterity/skills and motivation, in brushing at least once a day as soon as the teeth start erupting. As soon as practical, the child should be given responsibility to brush the second time.

- Introduction of fluoride is essential for primary and mixed dentition.

- In children (under six years) there is a lack of evidence on the comparative effectiveness of power and manual toothbrushes.

- This consensus suggests that power brushing can be started at any age if parent and child are comfortable with it.

- Bluetooth enabled power brushes with interactive apps have the potential to aid in better compliance from children.

\section{What should be Recommended to Orthodontic Patients?}

In a meta-analysis, Kaklamanos and Kalfas ${ }^{12}$ identified only five studies suitable for data synthesis. They found that, based on quality assessment and the short experimental period of these trials, current evidence is insufficient to support the comparative efficacy of powered toothbrushes in reducing gingivitis in patients undergoing fixed orthodontic appliance therapy. In a study by Clerehugh et al., ${ }^{13}$ the group using the electric brush had significantly less interdental gingival bleeding, as determined by the Eastman interdental bleeding index both at week four and week eight, compared to baseline. However, when comparing the effectiveness of powered versus manual toothbrushes, no statistically significant difference was noted between the two groups.

In the absence of high-quality evidence, expert opinion regarding this question was offered by the panel. Therefore, it was advised:

- $\quad$ Based on the present data, orthodontic patients seem to benefit from both manual and power brushes. There is a lack of conclusive evidence of the clear benefit of one over the other.

- There is an increased need for interdental cleaning in orthodontic patients.

- Better designed studies of longer duration would be more clinically relevant in orthodontic patients.

- There was a consensus that there is a higher likelihood of enhancing compliance/convenience with the use of a powered brush.

\section{Is Power Brushing more Effective than Manual Brushing in the Prevention of Caries?}

The development of dental caries is influenced by several factors such as plaque control, sugar levels and frequency of exposure in the diet, and availability of regular exposure to fluorides. There are significant ethical considerations, which preclude allowing the development of caries in human studies, and so surrogate outcomes (most commonly plaque indices) are generally used in shortterm studies. It is thus not possible to draw evidencebased conclusions on this question. It is, however, the Delphi panel opinion that:

- Power brushing is more effective than manual brushing in the reduction of dental plaque.

- However, there is no clear evidence that this will lead to a reduction in the incidence of dental caries.

- Given the lack of evidence, there is a need for further research.

\section{Is Power Brushing more Effective than Manual Brushing in the Prevention of Gingivitis and Periodontitis?}

As noted above, Bosman and Powell ${ }^{7}$ showed that effective brushing once a day or every second day was sufficient to reverse gingivitis within 7 to 10 days, while Lang et al. ${ }^{8}$ again showed that effective brushing once a day or every second day prevented all clinical signs of gingivitis over a six week period. Since then, numerous short and several longer-term studies have confirmed the reversal of the clinical signs of gingivitis by use of both manual and power brushes. The panel was, however, unable to find any high-quality evidence that either form of tooth brushing was effective in the prevention or control of periodontitis. Consequently, the consensus was the following:

- While power brushes may have an impact on reducing/preventing gingivitis, the same cannot be said for periodontitis, as there is a lack of evidence to support this at present.

\section{Is Power Brushing more Effective than Manual Brushing in the Prevention of Peri-implant Diseases?}

Renvert et al. ${ }^{14}$ reviewed the literature on peri-implant diseases and concluded that while mechanical nonsurgical therapy (scaling and tooth brushing) could be effective in the treatment of peri-implant mucositis lesions, it was ineffective in controlling peri-implantitis. We were unable to identify any studies comparing manual and power brushes in the management of peri-implant diseases. Consequently: 
- There is no substantial evidence, which demonstrates the difference between power and manual brush efficacy towards the reduction of periimplant diseases.

- It seems reasonable to conclude that the greater efficiency of power brushes in plaque removal and control of gingivitis around teeth may have a similar benefit in reducing plaque-related periimplant mucositis.

- More research is needed in this important topic.

\section{Is Trauma more Likely with Power Brushes than Manual Brushes?}

Although concern has been expressed that power brushes may have the potential to cause damage to hard and soft oral tissues, this has been investigated extensively since the mid-1980s. Both Niemi et al. ${ }^{15}$ and a systematic review by Rajapakse et al. ${ }^{16}$ identified no evidence of any difference in tissue damage between manual and power brushes. It is indeed more likely that newer power brushes with a pressure feedback mechanism, and without a horizontal brushing movement are less likely to cause damage than inappropriately used manual brushes. The panel's conclusions were:

- There is no evidence to support the notion that power brushes are more harmful than manual brushes.

- Power brushes with an integrated pressure feedback mechanism may have the potential of reducing soft and hard tissue abrasion.

\section{DISCUSSION}

During the application of the Delphi procedure, the panel was very aware of the limitations of short-term studies, particularly where they used surrogate outcomes to draw major conclusions. Therefore, the panel concurred with the statement by Matthews ${ }^{17}$ "consensus is urgently required on the importance and validity of surrogate outcomes (e.g., plaque indices; changes in clinical probing depth) and their relationship to true outcomes (e.g., tooth loss and other patient-centered outcomes)". Ultimately patients are less concerned with indices, probing depths, furcation involvement and are more focused on freedom from pain, long-term tooth survival, acceptable aesthetics and effective function. Also, it was recognized that oral hygiene instruction is only part of the advice we should be offering patients. Although important, in the periodontal it is patient it is likely less crucial than smoking cessation counseling, or the need to receive 3 monthly professional, supportive periodontal care. With regard to caries prevention appropriate dietary advice and effective use of fluorides are at least equally important. However, it was beyond the scope of the Delphi conference to go into these areas in detail.

Furthermore, in investigating the questions addressed above it is apparent that several key pieces of information relating to tooth brushing do not have scientific evidence. It was, therefore, suggested that the following areas of research should be considered:

- There is a need for more research regarding patient compliance with tooth brushing advice. While it seems plausible that timers and Bluetooth ${ }^{\circledR}$ connected devices may motivate patients to better compliance, strong evidence is lacking, and this should be investigated.

- The limitation of most studies is the duration of the experimental period. By their nature, shortterm studies of 3 or 6 months use surrogate outcomes as measurements. Longer-term studies are necessary. Since fixed orthodontic appliance treatment may generally take 20 to 24 months to complete, it seems strange that most studies only last three to six months. Longer term studies using, where possible, real, patient-focused outcomes, are needed.

- Types of toothpastes used in conjunction with brushing need to be taken into consideration. While many studies compare manual and powered brushes with common toothpaste, there is little research assessing the effectiveness of power brushes used with a variety of toothpastes.

- Best time to brush requires more research. If we advocate one thorough clean per day, it is necessary to determine if morning brushing and evening brushing differ in terms of effectiveness.

\section{CLINICAL SIGNIFCANCE AND CONCLUSION}

Oral hygiene instruction and especially toothbrushing has been considered a fundamental part of the efforts to prevent caries and gingivitis. The participants of the Delphi panel agreed that twice daily brushing for two minutes and a systematic pattern is advisable. Moreover, they concluded that evidence suggests that powered brushes are safe and more effective in the short and long-term compared to manual brushes in terms of plaque removal and gingivitis reduction. For children and orthodontic patients, the likelihood of enhancing compliance/convenience by using of a powered brush was highlighted.

\section{ACKNOWLEDGMENT}

The Middle East Delphi Consensus Conference on Tooth brushing was supported by Procter and Gamble Gulf. 


\section{REFERENCES}

1. Linden GJ, Lyons A, Scannapieco FA. Periodontal systemic associations: review of the evidence. J Periodontol 2013;84(4 Suppl):S8-S19.

2. Berchier CE, Slot DE, Haps S, Van der Weijden GA. The efficacy of dental floss in addition to a toothbrush on plaque and parameters of gingival inflammation: a systematic review. Int J Dent Hyg 2008;6:304-314.

3. Hujoel PP, Cunha-Cruz J, Banting DW, Loesche WJ. Dental flossing and interproximal caries: a systematic review. J Dent Res 2006;85:298-305.

4. Sambunjak D, Nickerson JW, Poklepovic T, Johnson TM, Imai $\mathrm{P}$, Tugwell $\mathrm{P}$, Worthington HV. Flossing for the management of periodontal diseases and dental caries in adults. Cochrane Database Syst Rev 2001:CD008829.

5. Balasubramanian R, Agarwal D. Delphi Technique; A review. Int J Public Health Dent. 2012;3:16-25.

6. Kressin NR, Boehmer U, Nunn ME, Spiro A 3rd. Increased preventive practices lead to greater tooth retention. J Dent Res 2003;82:223-227.

7. Bosman CW, Powell RN. The reversal of localized experimental gingivitis. A comparison between mechanical toothbrushing procedures and a $0.2 \%$ chlorhexidine mouthrinse. J Clin Periodontol 1977;4:161-172.

8. Lang NP, Cumming BR, Löe H. Toothbrushing frequency as it relates to plaque development and gingival health. J Periodontol 1973;44:396-405.

9. Davies RM, Davies GM, Ellwood RP. Prevention. Part 4: Toothbrushing: what advice should be given to patients? Br Dent J 2003;195:135-141.
10. Yaacob M, Worthington HV, Deacon SA, Deery C, Walmsley A, Robinson PG, Glenny A. Powered versus manual toothbrushing for oral health. Cochrane Database Syst Rev 2014:CD002281.

11. Gibson S, Williams S. Dental caries in pre-school children: associations with social class, toothbrushing habit and consumption of sugars and sugar-containing foods further analysis of data from the National Diet and Nutrition Survey of Children Aged 1.5-4.5 Years. Caries Res 1999;33:101-113.

12. Kaklamanos EG, Kalfas S. Meta-analysis on the effectiveness of powered toothbrushes for orthodontic patients. Am J Orthod Dentofac Orthop 2008;133:187.e1-187.e14.

13. Clerehugh V, Williams P, Shaw WC, Worthington HV, Warren P. A practice-based randomized controlled trial of the efficacy of an electric and a manual toothbrush on gingival health in patients with fixed orthodontic appliances. J Dent 1998;26:633-639.

14. Renvert S, Roos-Jansåker A-M, Claffey N. Non-surgical treatment of peri-implant mucositis and peri-implantitis: a literature review. J Clin Periodontol 1998;35:305-315.

15. Niemi M-L, Ainamo J, Etemadzadeh H. Gingival abrasion and plaque removal with manual versus electric toothbrushing. J Clin Periodontol 1986;13:709-713.

16. Rajapakse PS, McCracken GI, Gwynnett E, Steen ND, Guentsch A, Heasman P. Does tooth brushing influence the development and progression of non-inflammatory gingival recession? A systematic review. J Clin Periodontol 2007;34:1046-1061.

17. Matthews DC. Prevention and treatment of periodontal diseases in primary care. Evid Based Dent 2014;15: 68-69. 